\title{
THE NERVOUS SYSTEM IN SARCOIDOSIS
}

\author{
By Michael Jefferson, D.M., M.A., B.Sc., M.R.C.P. \\ Consultant Neurologist, Queen Elizabeth Hospital, Birmingham
}

\section{Introduction}

In a historical sense sarcoid is a patchwork affair, its different clinical forms having only been put together slowly to make the clinical entity as it is recognized today. The skin lesions were first described by Jonathan Hutchinson (1875), who called it 'Mortimer's malady' after the name of his original patient. They were later described independently by Besnier (1889) and Boeck (1899), and, though the latter gave it its present name and remarked on lymph gland enlargement in it, it was left to Schaumann (1914 et seq.) to appreciate and emphasize the diffuse systemic nature of the disease. It is only in the last 20 years that the concept of sarcoidosis has been still further widened to include the subacute uveoparotitis of Heerfordt (1909), largely through the work of Bruins-Slot and his colleagues (1936, 1938), Pautrier (1937) and Longcope and Pierson (1937).

\section{Neurological Manifestations}

Sarcoidosis is not a common condition, though its incidence is certainly higher than was once supposed, and affection of the nervous system is a feature in only a small proportion of cases, though they are now a well-documented occurrence. The pattern of neurological symptomatology is variable, but it is possible to recognize three essential categories: the first where damage falls on the cranial and peripheral nerves, the second where the lesions are in the brain and neuraxis, and a third where the brunt is borne by the meninges. Many references to the literature are contained in recent review articles describing personal cases (Colover, 1948; Pennell, 1951; Jefferson, 1952, 1957; Aszkanazy, 1952; Meyer et al., 1953).

\section{Cranial and Peripheral Neuropathy}

Examples in this group are particularly liable to be associated with uveoparotitis as the presenting malady. Affection of one or both optic nerves, with actual swelling of the discs if the lesions are close to their insertion into the retinae, are not uncommon more or less simultaneously with sarcoid iridocyclitis, as are abnormalities of pupillary response from cycloplegia. There may also be external ocular palsies, ptosis, trigeminal sensory loss, facial palsy, loss of taste, nerve deafness and paralysis of palate, pharynx, vocal cords or tongue. Any one of these may appear alone or in combination with others to give a constellation of cranial polyneuropathy, and, as they are usually transient, there may be a sequence of nerve pareses with some clearing as others come on. Paralysis of the seventh nerve is probably the commonest of all and, though it is often contemporaneous with parotid swelling, it can occur in its total absence. Its onset can be as abrupt as an ordinary Bell's palsy, but it may be more lethargic, taking several days to become complete. Quite often it is bilateral either more or less simultaneously, or with only an interval of a few weeks between attack on one side and the other; in such cases sarcoid aetiology should always be suspected, because other causes of acute facial diplegia are so rare.

Signs and symptoms of peripheral nerve dysfunction may also appear by themselves or in company with, or before or after, cranial nerve troubles. The clinical picture is not constant, but pain is a common characteristic whatever the exact presentation. There may be weakness and blunting of sensory acuity affecting all modes, more or less symmetrical in all four limbs and have a predominantly distal distribution, resembling the pattern seen in acute infectious polyneuritis, but the onset is apt to be more ingravescent. However, asymmetrical forms suggesting lesions of a congeries of isolated nerve roots or a selection of peripheral nerve trunks (or a mixture of the two) are more usual and, as with the cranial neuropathies, there is apt to be some scatter in point of their time of appearance. The tendon reflexes are depressed or abolished and there may be girdle pains and sensory deficit on the body from involvement of intercostal nerve branches.

Recovery from cranial and peripheral neuropathy in a matter of weeks or a few months is the rule, but it is not always quite complete and there can be later relapse. 


\section{Central Nervous Involvement}

Sarcoid disease of the central nervous system may take many forms and, unlike the peripheral group, as a general rule it bears no particular relationship to the uveoparotid variety. It may declare itself by signs of cerebral hemisphere disorder, with generalized or focal epilepsy, motor or sensory hemiplegia, hemianopia, dysphasia, personality change or mental confusion. In some instances the condition remains static once established, but in others it is progressive, with headache and papilloedema from steady increase in size of the causal sarcoid granuloma, and so simulates neoplasm in the brain. Or the lesion may be deep, so that (rarely) the basal ganglia are affected, with production of a quasi-Parkinsonian syndrome. The hypothalamus and pituitary region is a particularly favoured site of attack and results in diabetes insipidus, obesity, loss of sexual potency and amenorrhoea, with or without field defect from coincident involvement of optic chiasma or tracts. Polyuria and polydipsia recovering spontaneously have been recorded a number of times (an example was included amongst Heerfordt's original cases), where a hypothalamic sarcoid lesion which cleared later can be presumed, but, as with the hemisphere lesion, a chronic or progressive course suggestive of tumour in that neighbourhood is at least as, or more, common.

As well as producing supra-tentorial symptomatology, sarcoid may affect the posterior fossa to produce cerebellar dysfunction with nystagmus, ataxia and dysarthria, and again tumour can be mimicked if the lesion grows in size. Alternatively, the brain stem can be primarily implicated to suggest such conditions as disseminated sclerosis, intrinsic angioma or glioma. Lastly, it can appear in the guise of spinal cord disease, which can be mistaken for a degenerative affection or compression by extrinsic or intrinsic new growth, the neurological semeiology depending on the level and extent of damage.

On the whole, sarcoidosis of the central nervous system is sinister in its effects, because it does not often show the evanescence which marks its peripheral nervous lesions and the deficits it causes are permanent.

\section{Meningeal Affection}

Implication of the meningeal coverings of the brain and spinal cord by sarcoid disease happens occasionally. Frank meningitis, with neck and spinal stiffness and restriction of straight leg raising, is a rarity, though there are some cases on record, of subacute or chronic type, in most of which signs of simultaneous nervous mischief have also been present. However, evidence of latent meningitis is fairly frequent in patients with neuro- logical sarcoid lesions, as witnessed by increase in the cerebrospinal fluid protein, mild lymphocytic pleocytosis and (rarely) reduction in sugar and chloride content. The meningeal lesions themselves may be small and multiple, or solitary granulomata which may reach some size. The latter seem to have some preferential affinity for the posterior fossa and may come to fill and obstruct the fourth ventricle.

\section{Mixed Forms}

In some cases it is clear that the central and peripheral nervous systems can both be affected in sarcoidosis, either at the same time or in separate episodes of the disease.

\section{Pathology and Aetiology}

The pathology of sarcoid lesions, whether they affect cranial or peripheral nerves, brain or spinal cord, or meninges, is strictly comparable with their histological appearance in other tissues. The basic feature is the follicle, which appears to the naked eye as a tiny grey or yellowish granule. Microscopically, it consists of large pale staining epitheloid cells, which may be in concentric or whorl formation in larger foci, but in small ones show no particular arrangement. In long-standing lesions the central area may show necrosis, which if variously termed ' fibrinoid ' or 'paramyloid', buk there is no caseation. Giant cells are usually conspicuous elements, though they may be absent from some follicles; quite frequently they contain intracytoplasmic inclusions, either asteroid or of deeply staining lamellated form. Lymphocytes are usual in peripheral situation round the follicle, and plasma cells are occasional. In large granulomata the structure is one of conglomeration of myriads of follicles, often with much interstitial fibrosis. Healing of follicles takes place by sclerosis, starting marginally and advancing centripetally, and in this manner acellular areas of hyaline material are produced, leaving only traces here and there of the original follicular structure.

The aetiology of sarcoidosis is still not established and the incidence of nervous disorder throws no light on it. The main theories are (I) that it is a modified (anergic) form of tuberculosis, (2) that it is due to some other infectious agent, bacterial, viral or protozoal, as yet unidentified, and (3) that it is not an aetiologically pure condition at all, but a form of systematized tissue reaction which may have many causes. The tuberculous hypothesis, which has been lately in decline of popularity, has gained new support from the finding of chemical residues in sarcoid lesions, which strongly suggests that tubercle bacilli have been present in them at one stage (Nethercott and Strawbridge, 1956). 


\section{Diagnosis and Treatment}

The diagnosis of sarcoidosis of the nervous system is not difficult if other symptoms and signs of sarcoid affection are there to give the clue, such as swollen salivary glands or superficial lymph nodes, cutaneous eruptions, uveitis, or chest symptoms with radiographic signs. The latter are frequently present with cranial and peripheral nerve manifestations or are readily discernible in the past medical history. Unfortunately, with lesions of the central nervous system more often than not they are absent when the patient is seen and previous illness suggestive of sarcoidosis may not be obtained in anamnesis or, if recalled, may be hard to evaluate. In these circumstances correct diagnosis becomes a most formidable matter, for the neurological symptoms themselves only bespeak the anatomical site of the disease and convey no intimation of pathology beyond what can be inferred from their manner of onset and course. In fact, it is usually made only after extensive investigation, ending with histological examination of biopsy material taken at craniotomy or laminectomy or at autopsy.

Corticoids are now fashionable in medical treat- ment of sarcoidosis and have more or less replaced calciferol, which had adherents before, though it is doubtful if they have any more influence on the natural history of the disease than had earlier remedies. Their trial may be justified in sarcoid neuropathies, but they are certainly useless against most central nervous lesions, which always demand surgery when they are acting in a space-taking way.

\section{BIBLIOGRAPHY}

$\checkmark$ ASZKANAZY, C. L. (1952), f. Neuropath., II, 392. BESNIER, E. (1889), Ann. Derm. Syph. (Paris), 10, 333. BOECK, C. (1899), f. cutan. Dis., 17, 543.

BRUINS-SLOT, W. J. (1936), Ned. T. Geneesk., 80, 2859.

BRUINS-SLOT, w. J., GOEDBLOED, J., and GOSLINGS, J (1938), Acta med. scand., 94, 74.

COLOVER, J. (1948), Brain, 71, 45 I.

HEERFORDT, C. F. (1909), v. Graefes Arch. Ophthal., 70, 254.

HUTCHINSON, J. (1875), 'Illustrations of Clinical Surgery', London, J. A. Churchill.

JEFFERSON, M. (1952), Brit. med. F., ii, 916.

JEFFERSON, M. (1957), Brain (in press).

- LONGCOPE, W. T., and PIERSON, J. W. (1937), Bull. fohns Hopk. Hosp., 60, 223.

MEYER, J. S., FOLEY, J. M., and CAMPAGNA-PINTO, D. (1953), Arch. Neurol. Psychiat. (Chicago), 69, 587.

NETHERCOTT, S. E., and STRAWBRIDGE, W. G. (1956), Lancet, ii, 1132.

PAUTRIER, L. M. (1937), Bull. Soc. méd. Hóp. Paris, 53, 1608.

PENNELL, W. H. (1951), Arch. Neurol. Psychiat. (Chicago), 66, 728

SCHAUMANN, J. (1914), 'Sur le Lupus Pernio', Stockholm, P. A. Nordstedt, 1934 .

\title{
In gastro-intestinal conditions...
}

\author{
When solid food is out of the question - in diarrhoea due to food poison- \\ ing or other intestinal infection - frequent sips of Lucozade, so easily \\ assimilated, will do much to maintain the patient's strength and morale. \\ Lucozade has all the qualities of glucose combined with a refreshing \\ palatability that makes it acceptable in all circumstances of weakness \\ and exhaustion.
}

Lucozade is supplied in $6 \mathrm{oz}$. and $26 \mathrm{oz}$. bottles. It is lightly carbonated with an attractive golden colour and a pleasant citrus flavour. It contains $23.5 \% \mathrm{w} / \mathrm{v}$ Liquid Glucose B.P., and its energy value is 21 Calories per fluid ounce.

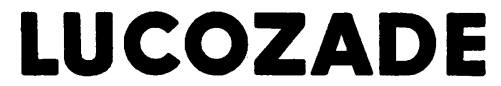

